\title{
Android-based non-contact elevator overspeed governor speed measuring system
}

\author{
Xu Chen ${ }^{1, a}$,Xiangdong $\mathrm{Li}^{2, \mathrm{~b}}$
}

${ }^{1} \mathrm{NO} .107$,longjiang building, caochangmen street, gulou district, nanjing city, jiangsu province,China

${ }^{2}$ NO.107,longjiang building, caochangmen street, gulou district, nanjing city, jiangsu province,China

accube1984@163.com, 'Ixd1963@163.com

\begin{abstract}
Keywords: Elevator overspeed governor, Rotational speed measurement, OpenCV, Android. Abstract.With the rapid development of technology, intelligent mobile phone hardware performance improving. Meanwhile, the openness of Android system is also conducive to the development of software.Therefore this paper proposes a elevator overspeed governor speed measuring system based on Android Smartphone, using the mobile phone camera and OpenCV image processing technology for non-contact speed measurement, it not only reduces the existing test instruments volume and production cost, but also increases its reliability.
\end{abstract}

\section{Introduction}

Elevator speed limiter is one of the important safety parts of the elevator, when the elevator operation of the car for various reasons to occur when speeding or even fall can make the elevator safety stop. So the elevator speed limiter need to be checked regularly to verify.its performance is reliable. Currently, the elevator speed limiter is mainly through the elevator speed limiter calibration instrument. It by driving the elevator speed limiter roulette, to its simulation movement speed.Test record at this time of the movement speed, compared with the rated speed, can judge whether the speed limiter functions in good condition. Visible, the speed limiter wheel speed detection, is the key to the whole instrument.

\section{Speed measuring method of traditional elevator speed limiter}

At present, the speed measurement methods of the speed limit device of the elevator include contact measurement and non-contact measurement.

Contact speed measurement methods need to be speed measuring device with rotating speed wheel direct contact with the measured speed. Common types include speed wheel and encoder type two kinds.

Non-contact speed measurement methods should first marker on the wheel speed limit preset, through calculating unit time read marker number calculates the speed sensor.According to different sensors, the common hall type and photoelectric type two kinds.

However, the existing elevator speed limiter calibration instrument no matter what kind of measurement method, all need independent sensor device, so as to lead to the volume of the instrument becomes greater.With the rapid development of the embedded operating system and the hardware of the mobile intelligent terminal, the test instrument system based on the mobile terminal will gradually become a developing trend. This paper proposes a video speed measuring method based on Android smartphones, via cell phone cameras can detect speed without the need for other devices. Has the advantages of small size, convenient operation, anti-interference, etc..

\section{Video speed measuring principle}

Video speed measuring method for rotating object, the usual approach is to refine the image to be a mark line, by calculating the angle difference and time difference of the mark line in the two frame 
picture, the speed of the object is obtained. This method has the advantages of high measurement accuracy, but the algorithm is complex, has certain requirements of mobile hardware.

Due to the low general speed elevator speed limiter, this paper proposes a simplified video speed measuring method. The principle base on the non-contact photoelectric measuring method of rotational speed of similar, wheel speed limit in the upper part of post some reflective logo, mobile phone camera capture speed round rotating multiple pictures, by comparing to the adjacent two photos to judge whether to revolve, accumulated per unit time by the number can be calculated RPM (revolutions per minute).Therefore, this system is mainly divided into frame image acquisition, image processing, calculation speed and other steps, the system function module is shown in Fig.1.

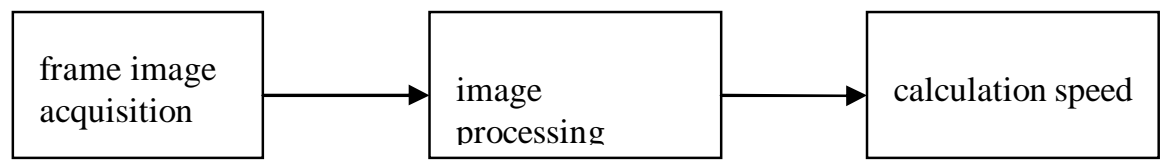

Fig.1 System function module

\section{Software design}

The main functions of the system software is to obtain the image, image processing and calculation speed.Software development environment: Java SDK+ Eclipse +ADT+CDT+OpenCV 2.4.4 + Android SDK.

Video processing involves a large number of complex algorithms, which will increase the difficulty and workload of software development. OpenCV is a class of $\mathrm{C}$ and $\mathrm{C}++$ language source code files, these source code files to achieve a number of commonly used computer vision algorithm. So in the software development just calls the corresponding source code, and no longer need to write code to achieve the function. This greatly reduces the difficulty of the preparation of software, especially since the 2.3 version of the OpenCV Android platform for the Java interface to facilitate the development and use of Android.

Image acquisition. Image acquisition is the actual access to the application of Camera Service. Through it can handle the data captured by the camera. It can be set by the Android list file in the call camera hardware and SD card read and write permissions. The code would look like the following.

Camera permissions:

<uses-permission android:name="android.permission.CAMERA"/>

SD card read and write permissions:

<uses-permission

android:name="android.permission.MOUNT_UNMOUNT_FILESYSTEMS" />

<uses-permission

android:name="android.permission.WRITE_EXTERNAL_STORAGE"/>

In order to reduce the influence of operating personnel manual, the display area was designed the viewfinder frame layer for reference of the tester shown in Fig. 2, to reduce the artificial shaking while operating.

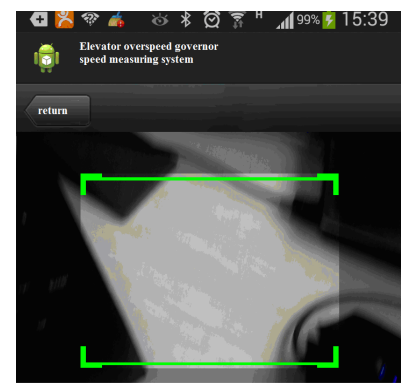

Fig.2 The image acquisition interface 
The image processing. The first is the image gray scale processing. The simplest way is to use "CV_LOAD_IMAGE_GRAYSCALE" function, it can be directly in loading the image processing, the resulting image is a single channel. This code is shown below.

IplImage* Igray=cvLoadImage("test.jpg",CV_LOAD_IMAGE_GRAYSCALE)

The second step is image binarization processing, image binarization is an important technology in image preprocessing, which set a certain threshold of the gray level image pixels into is greater than the threshold value of pixels and less than the threshold value of the pixel group of two parts. Its function is:

$$
g(x, y)=\left\{\begin{array}{ll}
0 & f(x, y)<\text { threshold } \\
255 & f(x, y) \geq \text { threshold }
\end{array}\right\}
$$

Thus, binarization image processing is gray image point set as 0 or 255, that is the whole image shows the obvious effect of black and white. This image set only with pixel value of 0 or 255 points, no longer involves the pixels of multistage value. So the process becomes simple. OpenCV provides a function " CV Threshold " for the use of a single channel array of fixed threshold operation, you can threshold operation of the gray image to get the two value image. The threshold is set to 254, as shown in Fig3 in the actual test.

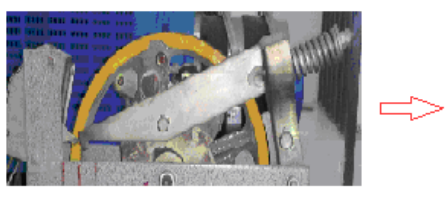

original image

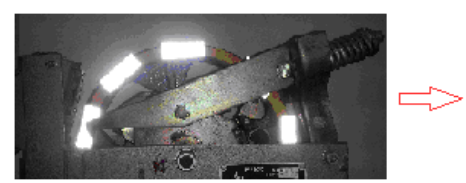

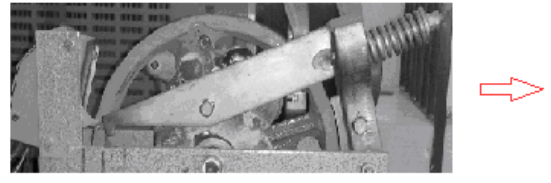

gray scale processing

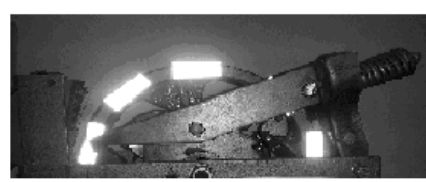

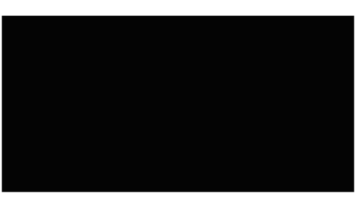

binarization processing

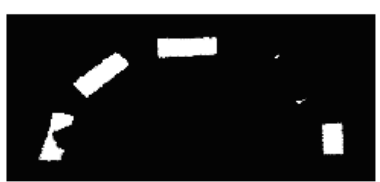

Fig.3 The image processing

Speed calculation. Speed measurement accuracy are inseparable with the number of frames, in theory, the more the number of images, the more accurate. However, due to the phone's hardware configuration restrictions, a large number of pictures will lead to the processing time is too long. At the same time, the general mobile phone video frames between 25 to 30 frames per second, so it take the number of frame graphics for 50 pieces of picture processing. Before calculating the speed need to handle 50 pictures, because the speed limit on the wheel attached to the reflective logo, after the image processing in front of the formation of a distinct black and white picture. When two adjacent frames are judged to be inconsistent, the meter is 1 circle. The program flow chart is shown in Fig.4. 


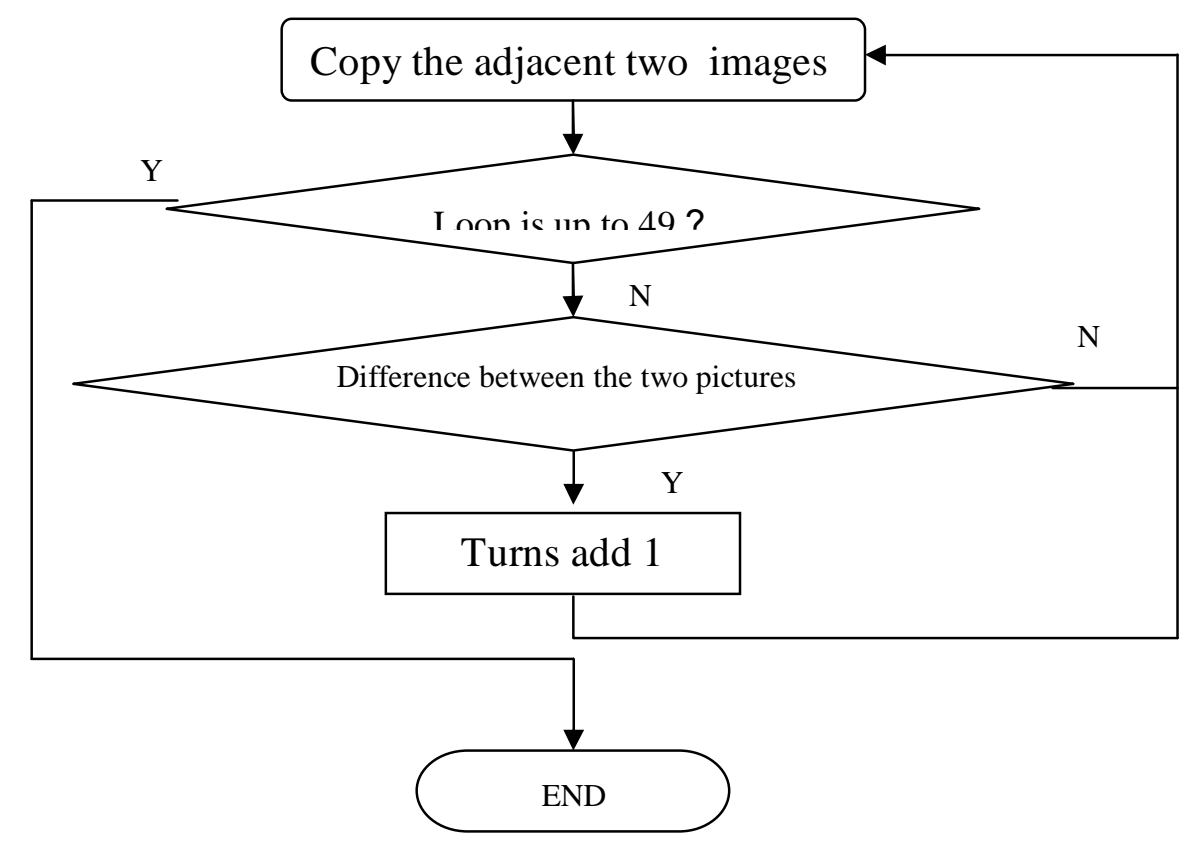

Fig.4 The program flow chart

Two adjacent frames can be judged by the function "CVAbsDiff" to do the differential operation, as follows.

IplImage* pre=NULL;

IplImage* curr=NULL;

IplImage* diff=NULL;

int Num=0;

while (curr=cvQueryFrame(pCapture))

\{

Num++;

if(NUm==1)

\{

next=cvCreateImage (cvGetSize(curr),8,3);

diff $=$ cvCreateImage $($ cvGetSize(curr), 8,3);

cvCopyImage(curr,next);

\}

else

\{

cvAbsDiff(curr,next,diff);

cvCopyImage(curr,next);

\}

\}

The final software testing interface as shown in Fig.5.

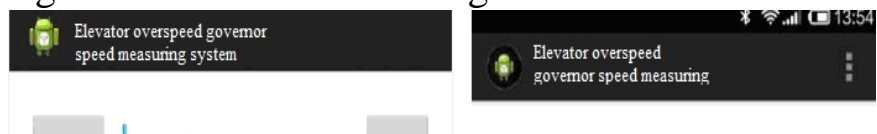

Serial number VIEW

Speed measurement

RPM $03 \mathrm{r} / \mathrm{s}$

Fig.5 Software testing interface 


\section{Conclusions}

In this paper, the OpenCV image processing technology for Android mobile phone elevator speed measurement method of the speed measurement method was studied and discussed, the mobile terminal and speed measurement equipment is proposed to combine the idea of. Combined with other features on the Android phone, greatly reducing the current elevator speed limit device calibration equipment volume, but also reduce the cost of procurement.

However, due to the limitations of mobile phone hardware and software is not perfect, the current system also has a low accuracy, can not measure a series of high speed and other issues, the need to further improve the improvement.

\section{References}

[1] Howse, J: Android Application Programming with OpenCV (Packt Publishing, England 2013).

[2] Kazimierz Orlowski, Jakub Sandak, Chiaki Tanaka. The Critical Rotational Speed Of Circular Saw: Simple Measurement Method And Its Practical Implementations. Journal Of Wood Science, Vol. 53(5)(2007),p.388-393.

[3] Ke Peng, Nianxiang Peng, Yanhong Chen: Computing Technology and Automation,Vol.29(1) (2010),p.76-79. (in Chinese)

[4] Jiecheng Meng, Hong Lv, Yunsheng Deng, Defang Liu : Control \& Automation, Vol.08 (2012),p.155-156 (in Chinese)

[5] Yongli Huang, Xiao Wang, Meiyun Kong: Android application development complete study manual(Tsinghua University press, China 2014). (in Chinese) 